\title{
Histoire du manuscrit médiéval, transmission des textes, des chants, compositions peintes et décors.
}

Le manuscrit 1 de la Bibliothèque municipale de Semur-en-Auxois au miroir de ses manuscrits « voisins »

Eduardo Henrik Aubert, Eliana Magnani et Daniel Russo

\section{(c) OpenEdition}

Édition électronique

URL : https://journals.openedition.org/cem/11055

DOI : 10.4000/cem.11055

ISSN : 1954-3093

Éditeur

Centre d'études médiévales Saint-Germain d'Auxerre

Édition imprimée

Date de publication : 15 août 2009

Pagination : 143-155

ISSN : $1623-5770$

\section{Référence électronique}

Eduardo Henrik Aubert, Eliana Magnani et Daniel Russo, " Histoire du manuscrit médiéval, transmission des textes, des chants, compositions peintes et décors. », Bulletin du centre d'études médiévales d'Auxerre / BUCEMA [En ligne], 13 | 2009, mis en ligne le 18 octobre 2010, consulté le 22 septembre 2022. URL : http://journals.openedition.org/cem/11055 ; DOl : https://doi.org/10.4000/ cem. 11055

Ce document a été généré automatiquement le 22 septembre 2022.

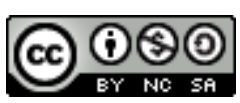

Creative Commons - Attribution - Pas d'Utilisation Commerciale - Partage dans les Mêmes Conditions 4.0 International - CC BY-NC-SA 4.0

https://creativecommons.org/licenses/by-nc-sa/4.0/ 


\section{Histoire du manuscrit médiéval, transmission des textes, des chants, compositions peintes et décors.}

Le manuscrit 1 de la Bibliothèque municipale de Semur-en-Auxois au miroir de ses manuscrits « voisins »

Eduardo Henrik Aubert, Eliana Magnani et Daniel Russo

Dans le cadre du programme pédagogique d'étude du manuscrit 1 de la Bibliothèque municipale de Semur-en-Auxois, provenant de l'abbaye de Saint-Jean de Réome (Moutiers-Saint-Jean) ${ }^{1}$, l'année écoulée a été l'occasion de s'intéresser, entre autres, aux manuscrits, qui, par leur typologie ou par les textes transcrits, viennent, soutenir compléter et élargir la réflexion en vue de la compréhension de la première partie du manuscrit de Réome (fol. 0r-69v). Le manuscrit 1 de Semur-en-Auxois peut être rangé parmi les libelli illustrés de vies de saints qui fleurissent en milieu monastique entre le milieu du $\mathrm{X}^{\mathrm{e}}$ siècle et la fin du XII ${ }^{\mathrm{e}}$ siècle ${ }^{2}$. En plus des lectures scandées par des lettrines enluminées, des homélies et des pièces liturgiques, dont certaines portent des neumes (fol. 45r-50r), pour l'office des fêtes de l'anniversaire et de la translation de saint Jean de Réome (fol. 0-50v) ${ }^{3}$, le manuscrit 1 contient la version $C$ de la vie du saint, d'après la classification de Bruno Krusch ${ }^{4}$ (BHL 4426). Plus d'une quinzaine de manuscrits médiévaux connus, pour la plupart des légendiers, contiennent des éléments du dossier hagiographique de Jean de Réome (BHL 4424 à 4431) ${ }^{5}$. Avec le manuscrit 1 de Semur, deux autres, au moins, possèdent des pièces liturgiques de l'office : le manuscrit 195 de la Bibliothèque municipale de Metz, provenant de SaintArnoul, et le Vaticanus Reginenses latinus493, fol. 105r-135v, réalisé au début du XI ${ }^{\mathrm{e}}$ siècle dans l'entourage de l'abbé Heldric (987/9-1010), réformateur clunisien et disciple de Maïeul, qui a dirigé les monastères de Flavigny, de Réome et de Saint-Germain d'Auxerre ${ }^{6}$.

Le manuscrit 195 de Metz daterait du XI ${ }^{\mathrm{e}}$ siècle, d'après Bruno Krusch, et il est réputé avoir été détruit depuis 1944. D'après la notice du catalogue de $1879^{7}$, il possédait, en ce qui concerne le dossier de Jean de Réome, des éléments textuels et graphiques le 
rapprochant du manuscrit 1 de Semur. Il « commence par cette inscription, en capitales disposées par lignes alternativement rouges et noires [...] : ANNO TERTIO REGNI DOMNI CLOTARII REGIS [...] », inscription dont la description et le contenu correspondent au texte et au dispositif graphique utilisé également au feuillet 50r-v du manuscrit 1 de Semur, et qui marque le début de la BHL 4426 (fig. 1 et 2). Toujours selon le catalogue, « après la légende, est cette rubrique : Hymnum ad Vesperas et l'hymne Sacra dies infunditur [...]. Et principatur monachis, etc. Suit l'office de S. Jean l'Hermite. Et ensuite : Incipit translatio corporis gloriosissimi Johannis confessoris Christi. Post primariam Johannis beatissimi sepulturam, etc. "Ces passages concordent avec les débuts de l'hymne transcrit au fol. 46v, lignes 1-4 (Sacra dies infunditur... principatur monachis), et du récit des translations copié au fol.30r (Post primariam Johannis beatissimi sepulturam),dans le manuscrit 1 de Semur. D'après B. Krusch, le manuscrit 195 de Metz était amputé d'un folio, et se terminait sur les mots calamitatibus christianorum, ce qui coïncide avec un passage du fol. $44 \mathrm{r}$ du Semur 1, et indique que le manuscrit de Metz contenait les BHL 4429 et 4430, c'est-à-dire, l'ensemble des translations et des miracles. Du point de vue des textes, ces passages du manuscrit 195 de Metz se retrouvent également dans le Vat. Reg. lat. 493, aux feuillets 124v (Anno tertio...), 121v (Sacra dies...) et 114r (Post primariam...), respectivement.

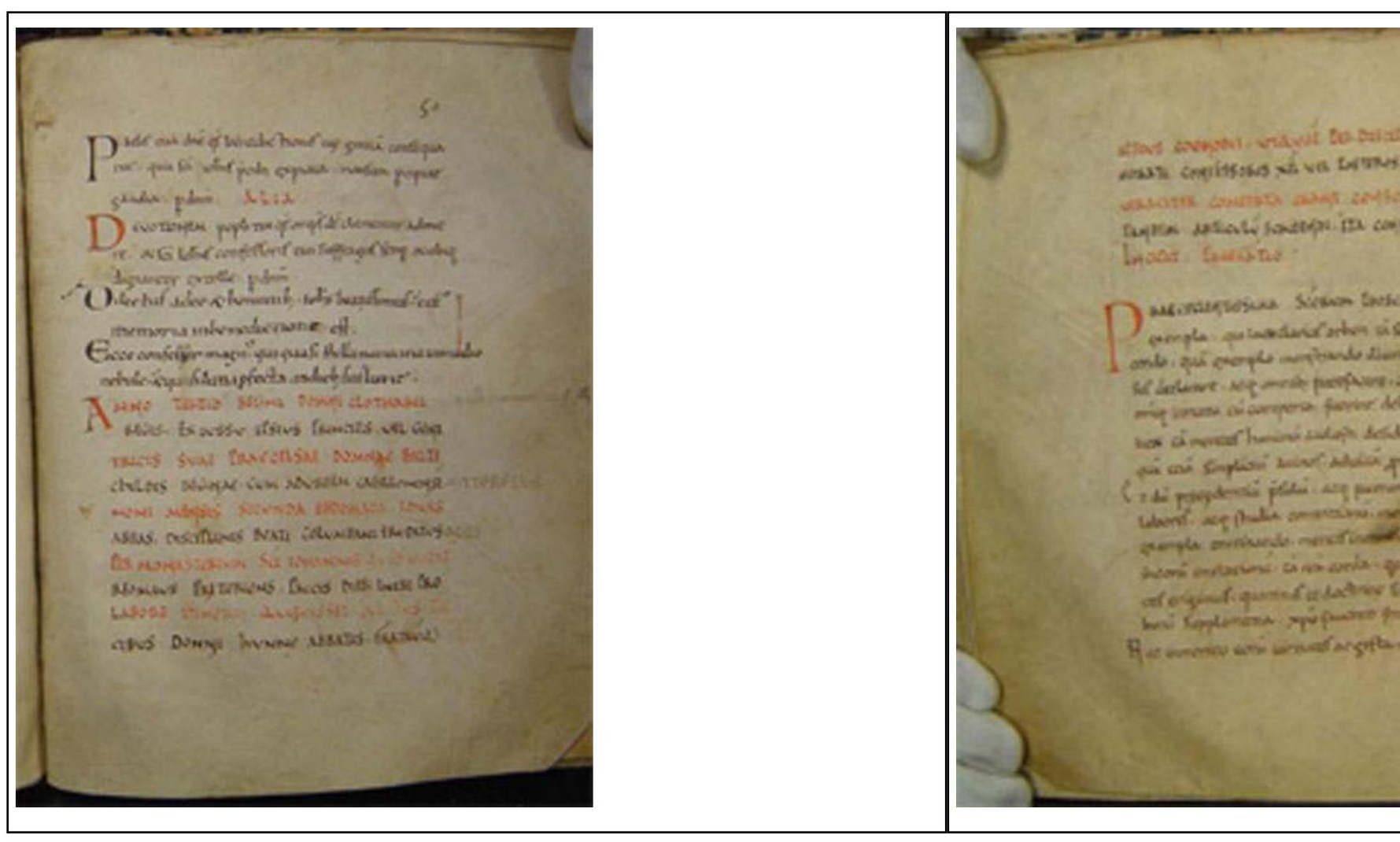

Fig. 1 et 2 - Semur-en-Auxois, Bibliothèque municipale, ms. 1, fol. 50r-50v. Début de la version $C$ de la Vie de saint Jean de Réome (BHL 4426), « en capitales disposées par lignes alternativement rouges et noires ».

Plusieurs indices tendent à confirmer que les feuillets 105r-135v du Vat. Reg. lat. 493, malgré leur originalité, ont été fortement inspirés du Semur 1. En attendant la réouverture de la consultation des manuscrits à la Biblioteca Vaticana, et sur la base 
seule d'une reproduction en microfilm, outre la corrélation textuelle (tableau I), on peut déjà avancer quelques observations qui vont dans ce sens, comme les lettrines qui marquent le découpage en lectures comme dans le Semur 1, ou l'espace blanc laissé au fol. 105v, qui semblait avoir été prévu pour l'exécution du Vir per cuncta, à la manière encore du Semur 1 (fol. 1r - fig. 3), si toutefois ceci se vérifie.

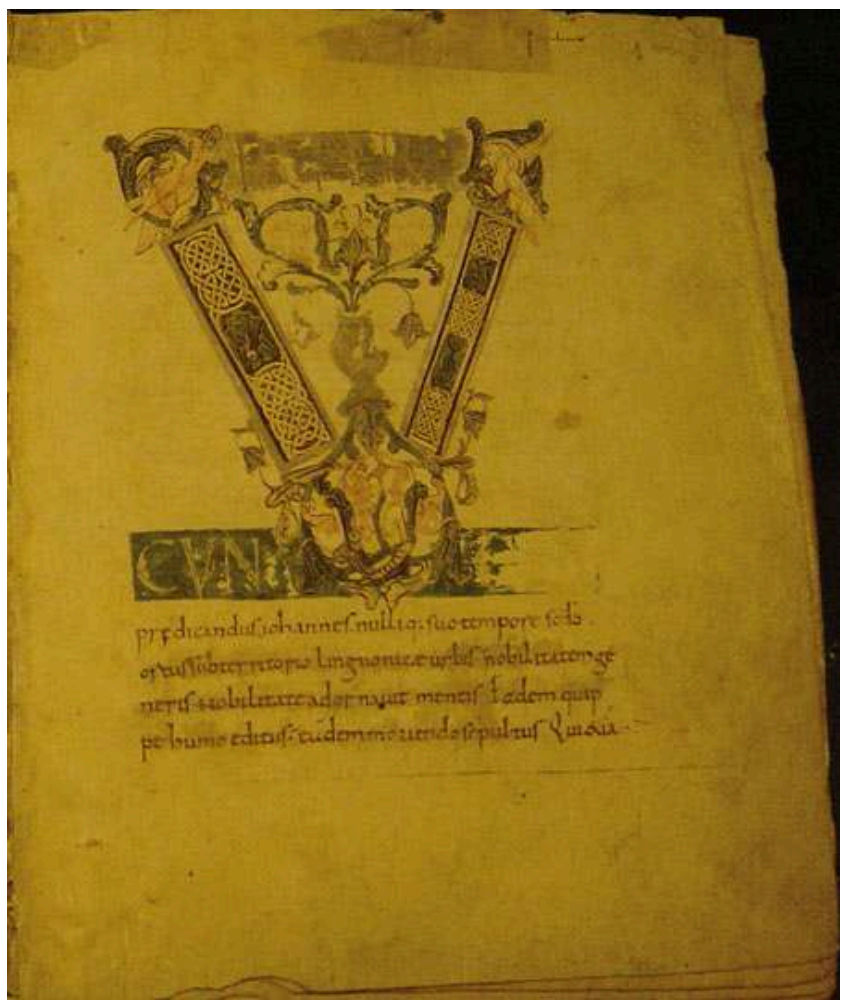

Fig. 3 - Semur-en-Auxois, Bibliothèque municipale, ms. 1, fol. 1r. Lectio I -Vir per cuncta (office de l'anniversaire de saint Jean de Réome ; BHL 4425).

La présence de neumes dans le Vat. Reg. lat. 493 (fol.121v-123r) permet aussi une comparaison avec le manuscrit 1 de Semur. Les deux témoins sont notés en neumes adiastématiques de type français (sans hauteur mélodique précise), mais la comparaison des écritures neumatiques doit se limiter à une portion de l'office de l'anniversaire : le Vat. Reg. lat. 493 ne contient pas les pièces du fol. $45 \mathrm{v}$ du manuscrit 1 de Semur - qui sont notées par deux mains distinctes de la main principale responsable des fol. 47r-49r - ni celles de l'office de la translation, où le texte a pourtant été copié; le troisième nocturne des matines de l'anniversaire n'offre que quelques incipit; les chants pour les laudes sont partiellement notés par des mains différentes de celle responsable des matines. Ces différences soulèvent le problème de la transformation de la liturgie dans sa transmission d'une communauté à une autre - il s'agit très clairement d'une transmission partielle et non intégrale des pièces de chant - et la distinction des mains suggère des moments distincts d'élaboration des offices dans chacun des deux manuscrits - l'inexistence de traces des pièces du fol. $45 \mathrm{v}$ du manuscrit de Semur dans le manuscrit de la Vaticane pourrait s'expliquer comme un ajout tardif, auquel les responsables de la copie du manuscrit de la Vaticane n'ont pas eu accès. Les différentes stratégies de copie posent aussi des questions sur la transmission liturgique: contrairement au manuscrit 1 de Semur, le scribe responsable de la plupart des neumes dans le Vat. Reg. lat. 493 a parfois essayé de 
placer plus haut les neumes qui correspondent à des sons plus aigus et plus bas ceux qui correspondent à des sons plus graves. S'agirait-il d'une aide supplémentaire dans la transmission d'un répertoire qu'on ne pratiquait pas dans la communauté pour laquelle le Vat. Reg. lat. 493 a été copié ?

D'autres éléments de comparaison viennent approfondir la compréhension du manuscrit 1 de Semur: ce sont les fragments de manuscrits de chant, qui, remployés comme reliures de livres modernes, se trouvent déposés aux Archives départementales de la Côte-d'Or, à Dijon, notamment dans la série B II 1104, où 25 des 41 fragments conservés proviennent de livres de chant. Ces témoins aident à placer le manuscrit 1 dans l'évolution des pratiques de l'écriture du chant dans la communauté de Réome. À ce titre, on pourrait signaler les deux fragments (liasses 1 et 41) qui proviennent d'un missel noté sans doute par l'une des mains qui est intervenue sur le fol. $45 \mathrm{v}$ du Semur 1 , ce qui signale la possibilité de comprendre les ajouts de ce feuillet comme faisant partie d'un investissement plus large de la communauté dans l'emploi (très précoce pour un missel) de l'écriture neumatique. L'un des 14 fragments d'un bréviaire du $\mathrm{XIII}^{\mathrm{e}}$ siècle (liasse 11) nous a fourni des repères aussi précieux qu'inattendus. Sauf cinq exceptions, les chants du manuscrit 1 de Semur ne se trouvaient que dans ce manuscrit et dans celui de la Vaticane et, étant notés en neumes adiastématiques, il était impossible de proposer une lecture mélodique de ces pièces. Or, le bifeuillet qui recouvre la liasse 11 de la série B II 1104 (fig. 4) contient des portions de l'office de l'anniversaire de saint Jean en notation carrée sur quatre lignes rouges, permettant une lecture mélodique, au moins partielle, de huit autres chants. Les renseignements fournis par ce précieux fragment ouvrent plusieurs pistes à la recherche : ils font mieux comprendre le sens de quelques signes neumatiques jusqu'alors énigmatiques; ils mettent en évidence les stratégies employées par les neumes adiastématiques pour communiquer le phénomène du chant et comment elles diffèrent de l'enregistrement en lignes ; ils montrent, enfin, quel a été le degré de transformation mélodique subi par ces pièces, à Réome, en deux cents, voire trois cents ans. Ainsi, par le recours à une documentation complémentaire, qu'elle soit conservée intégralement ou sous la forme de fragments, la compréhension des neumes du manuscrit 1 de Semur s'enrichit, tandis que leur rôle dans l'économie générale du manuscrit n'en ressort que mieux défini. 


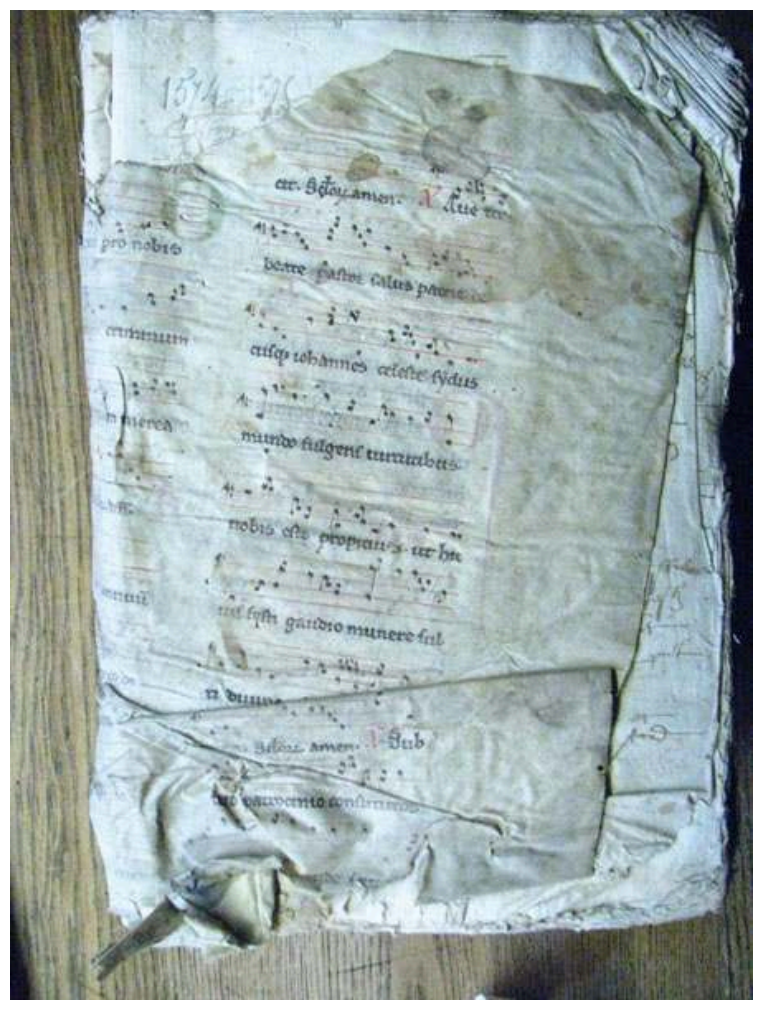

Fig. 4 - Dijon, Archives départementales de la Côte-d'Or, B II 1104, liasse 11. Fragment d'un bréviaire du XIII siècle, avec l'office de l'anniversaire de saint Jean de Réome, noté en notation carrée sur quatre lignes rouges.

La mise en parallèle de ces trois manuscrits suggère quelques observations d'ordre général. Ils sont d'abord les seuls à contenir les récits des translations et des miracles de Jean de Réome (BHL 4429 et 4430), ainsi que des hymnes et des pièces liturgiques, à la fois pour les offices et pour la messe. Ils posent la question de la relation entre l'organisation de l'office et la rédaction, ou la mise à jour, des translations et des miracles, par conséquent du contexte dans lequel ces manuscrits ont été réalisés entre le $\mathrm{X}^{\mathrm{e}}$ et le $\mathrm{XI}^{\mathrm{e}}$ siècle. Les trois manuscrits sont aussi les plus anciens connus qui renferment la BHL 4426, la version $C$ de la vie de Jean de Réome, dont l'élaboration est placée par $\mathrm{B}$. Krusch dans la première moitié $\mathrm{du} \mathrm{IX}^{\mathrm{e}}$ siècle, datation qui reste encore à confirmer. Cette version est, d'après les manuscrits répertoriés, la plus diffusée de la vie du saint - une dizaine de manuscrits entre le $\mathrm{XI}^{\mathrm{e}}$ et le $\mathrm{XV}^{\mathrm{e}}$ siècle. Il serait intéressant de pouvoir déterminer s'il existe un lien entre la diffusion de la BHL 4426 et l'effervescence de composition, liturgique, sonore, iconographique, dont témoigne le manuscrit 1 de Semur et le Vat. Reg. lat. 493.

Du double point de vue stylistique et artistique, l'étude a laissé voir une très forte originalité, cependant, du Semur 1, qu'il reste à mieux évaluer par rapport, notamment, au Vat. Reg. lat. 493, dans le groupe des manuscrits proches, mais aussi par rapport à celui des grands manuscrits liturgiques dans lequel il convient de l'insérer ${ }^{8}$. Dans les décors de ces manuscrits, les initiales notamment, des paquets de tiges végétales flexibles sont réunis, par endroits des lettres, sur les hastes et aux extrémités, tandis qu'à l'intérieur s'épanouit tout un réseau de spirales en fleurs - pampres, palmettes, feuilles d'acanthe -, qui forme un vocabulaire reconnu d'ornementation, bien souligné à l'époque, tout en courbes mêlées et intriquées les unes aux autres. De ce point de vue, 
le Vat. Reg. lat. 493 est également à situer dans cette mouvance. Seul l'examen attentif du manuscrit permettra, à l'avenir, d'avancer sur cette voie. De façon générale, domine encore l'idée d'entrecroiser les formes et les figures entre elles, en suggérant l'impression visuelle d'un prolongement presque infini, en dehors du cadre que constitue le feuillet, mais aussi au-delà de la portion d'espace réservé à l'initiale. Dans le Semur 1, nous observons, du reste, que les initiales aux animaux, donc à décor zoomorphe, forment une variante animée des initiales figurées, et qu'elles suggèrent le mouvement, l'exubérance parfois, qui fait du manuscrit un organisme toujours relié à son environnement de vie ${ }^{9}$.

À l'intérieur de la composition d'ensemble, dynamique, tout est coordonné, dans le Semur 1, chaque détail participant à la cohérence du feuillet, puis du cahier, enfin du livre tout entier même si, pourtant, tout y relève d'une composition nettement " acentrique ", pour le dire autrement, polycentrique (fig. $5,6,7)^{10}$. Tout commencement est en même temps inscrit comme recommencement. Se posent donc la question du continu dans le discontinu, puis celle de la mise en forme d'un cycle à travers les différentes lettres initiales de couleurs et de figures animales. Tout au long du texte, mais sans vraiment de rapports exacts constatés avec lui, se déroule une action, purement visuelle, de traits, de contours et de taches de couleurs, à l'issue de laquelle s'accomplit un effet d'images isolées ou, ce qui revient au même, une suggestion de petits tableaux à part, rattachés les uns aux autres par leurs seules propriétés internes : les qualités du dessin, de la forme, des matières colorantes tissent une trame unitaire et perceptible au regard, détachée du contenu textuel ou jouant, par rapport à celui-ci, un rôle plutôt lointain de marqueur, soulignant les points de passage et les lieux forts de la vie de saint Jean, sans plus. Hors de toute relation de cause à effet, c'est alors l'image visuelle qui est ainsi affirmée dans sa matérialité de chose fabriquée, ordonnée et produite avant tout pour être vue, perçue, jouée et rejouée par le regard. Nul doute que le prix de cette réalisation s'avère élevé, qu'un travail de grande maîtrise est nécessaire, et que tout ceci s'accompagne d'une longue accoutumance aux lettres figurées, à leur assemblage, à leur technique : le livre conçu pour supporter l'identité de la communauté monastique l'est encore pour témoigner, en soi, comme chose, de son très grand prestige à travers l'ancienneté de son fondateur et à un moment, sans doute, particulièrement fort de son histoire. 


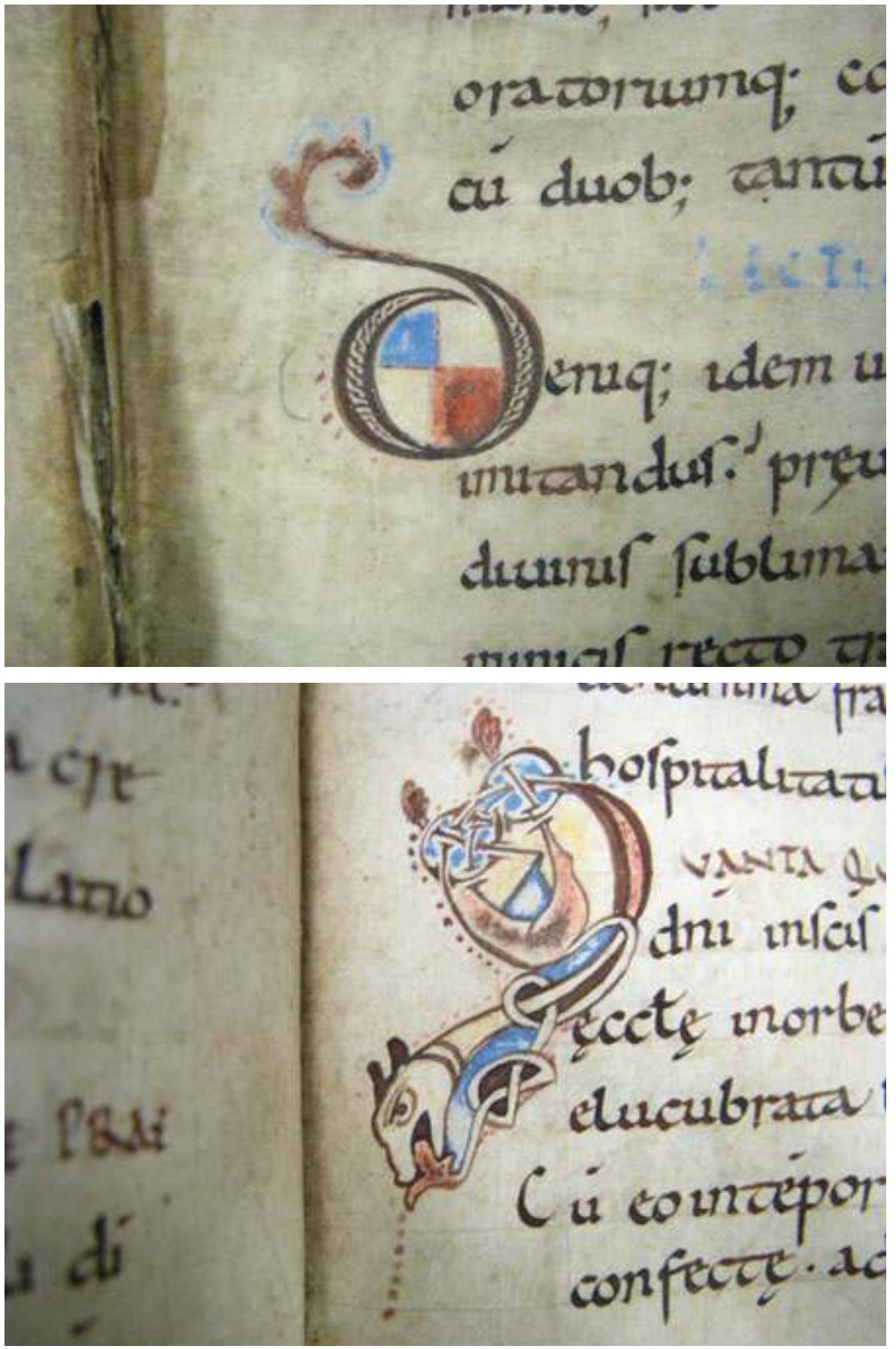




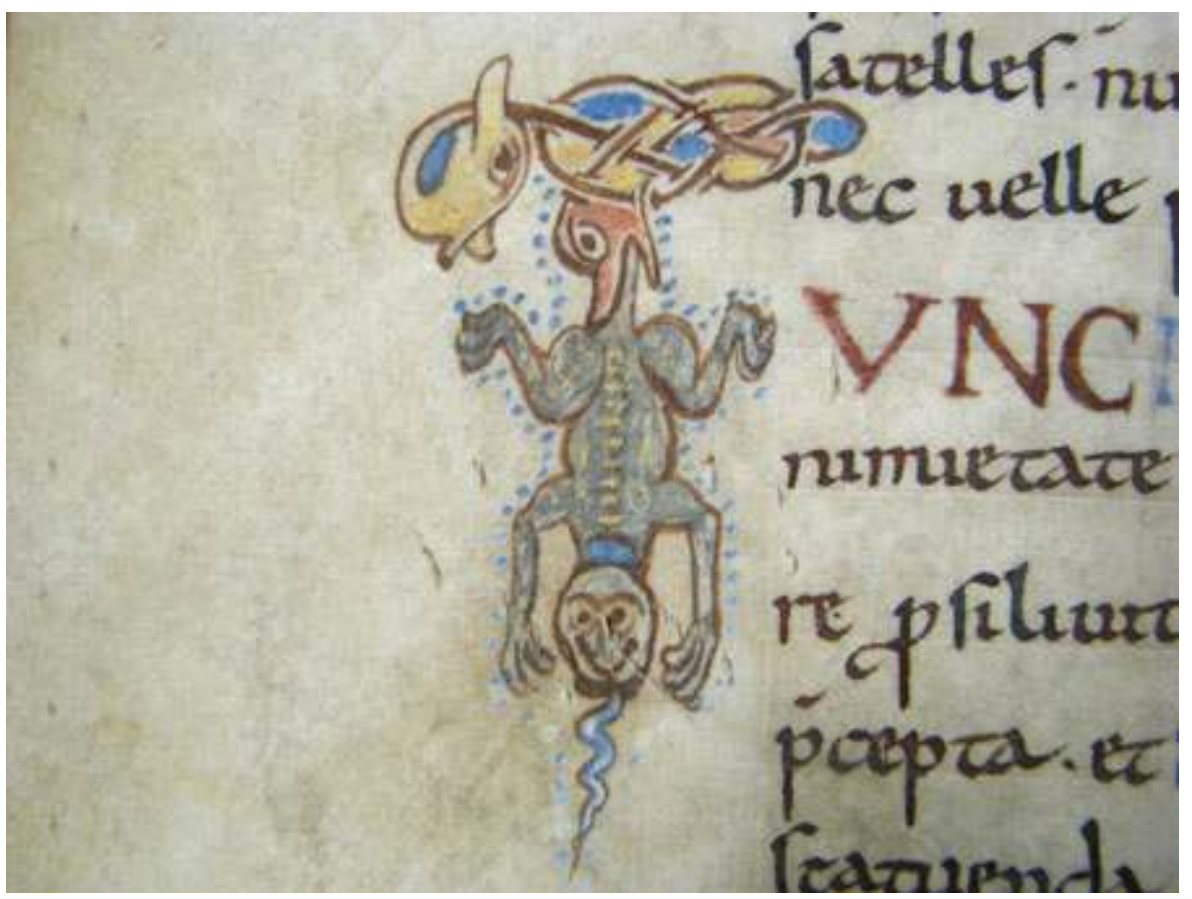

Fig. 5, 6 et 7 - Semur-en-Auxois, Bibliothèque municipale, ms. 1, fol. $2 \mathrm{r}$ $(D), 12 \mathrm{r}(Q), 22 \mathrm{v}(T)$.

Dans le Semur 1, les principes de la répétition et de la variation s'enchaînent l'un à l'autre sans aucune rupture de forme. Il est plus difficile, compte tenu des conditions de la consultation présente, d'analyser de même le Vat. Reg. lat. 493. Dans le cours de notre recherche, nous en resterons donc à la formulation de ces hypothèses sur un possible rapprochement. Nous constatons, toutefois, pour le Semur 1, que chaque reprise de forme et de couleur, de trait et de motif, est affichée comme une reprise, un retour en arrière, comme un rappel du point de départ et de l'origine, du début du livre et de ses décors, un signal vers les deux premières pages enluminées. Nous dirons que ces reprises agissent comme des retenues dans le champ plus large, plus étoffé, de l'événementiel hagiographique que le biographe rapporte de saint Jean et que, de cette manière, elles font renaître des séquences déjà perçues au regard : par ce procédé, on les replace sans cesse dans l'histoire vécue, mais aussi écrite, par la communauté des moines, dans son espace d'existence ${ }^{11}$. Il n'y a pas de progression en droit fil, ni continu, de l'intrigue visuelle; au contraire, bien plutôt un épanouissement simultané et discontinu d'une multiplicité de thèmes parallèles qu'on rapproche et met en relation formelle. La composition est plus musicale que discursive, faisant appel à une mémoire entrelacée des figures et des motifs, et à la reconnaissance finale de la présence d'une chose ainsi constituée comme le bien, en propre, du monastère. C'est sur cette voie que l'enquête devra être poursuivie pour le Semur 1 et pour les manuscrits qui peuvent lui être rapprochés. 


\section{ANNEXES}

Tableau I : Composition synoptique des manuscrits 1 de Semur-en-Auxois, Vat. Reg. lat. 493 et 195 de Metz

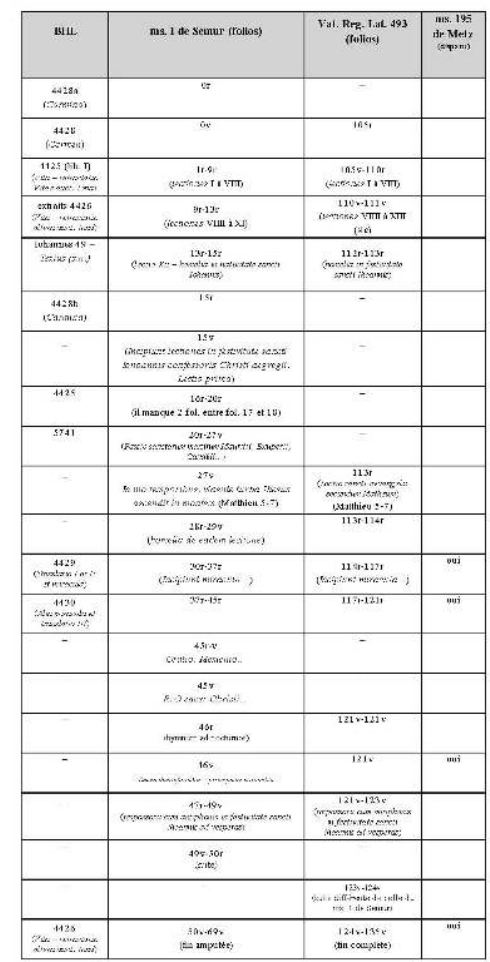

Tableau II : Les concordances pour les neumes du ms. 1 de Semur-en-Auxois dans le ms. Vat. Reg. lat. 493 et dans des manuscrits notés en lignes 


\begin{tabular}{|c|c|c|}
\hline 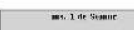 & 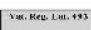 & 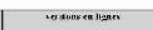 \\
\hline \multicolumn{3}{|c|}{ 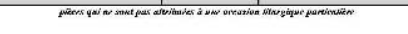 } \\
\hline Ro aserec Chant & & 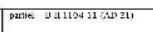 \\
\hline 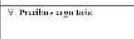 & & 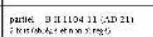 \\
\hline \multicolumn{3}{|l|}{ 2. Whiturats } \\
\hline \multicolumn{3}{|l|}{ 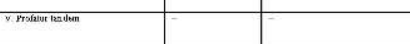 } \\
\hline \multirow{2}{*}{\multicolumn{3}{|c|}{ 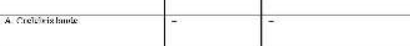 }} \\
\hline & & \\
\hline \multicolumn{3}{|l|}{ 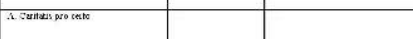 } \\
\hline \multicolumn{3}{|c|}{ 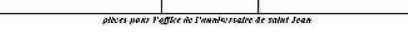 } \\
\hline \multicolumn{3}{|l|}{ reman } \\
\hline 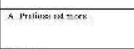 & smmplut & 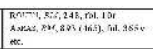 \\
\hline \multicolumn{3}{|l|}{ sestents } \\
\hline 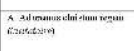 & somplate & 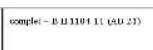 \\
\hline \multicolumn{3}{|l|}{ 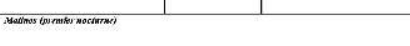 } \\
\hline 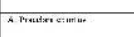 & stmenglis & F \\
\hline 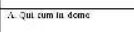 & semplite & \\
\hline 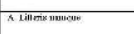 & semplente & \\
\hline 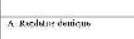 & smomplate & F \\
\hline 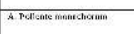 & Gmplis & \\
\hline A Resentis o a qud dem & complate & \\
\hline R. Hisest idnumer & smmplat & - \\
\hline P. Cum Froposthim & Samplis & \\
\hline 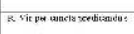 & somplet & \\
\hline 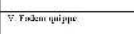 & \begin{tabular}{|c|c|c|} 
smmplsts \\
\end{tabular} & \\
\hline 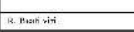 & sumplis & - \\
\hline
\end{tabular}

\begin{tabular}{|c|c|c|}
\hline 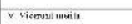 & somplester & \\
\hline 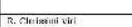 & & 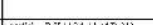 \\
\hline & monses & \\
\hline 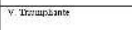 & somplet & \\
\hline \multicolumn{3}{|l|}{ 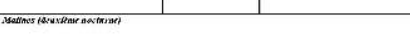 } \\
\hline 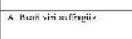 & smmplats & 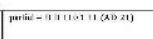 \\
\hline A Mesumat thestit & samplist & 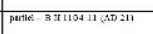 \\
\hline 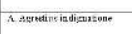 & semplete & 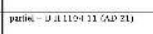 \\
\hline 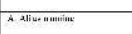 & \begin{tabular}{|l|} 
smmplus \\
\end{tabular} & 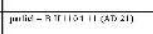 \\
\hline 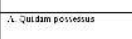 & somplet & 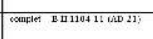 \\
\hline 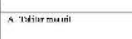 & stomplest & portih- - \\
\hline R. nedass peter & samplas & \\
\hline 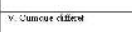 & semplite & \\
\hline 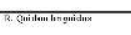 & 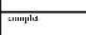 & \\
\hline 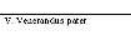 & somplite & \\
\hline 8. vilsashatas & somplict & 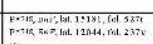 \\
\hline Y.U1 keseitus & somplet & \\
\hline 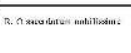 & 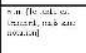 & 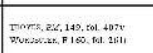 \\
\hline 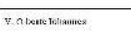 & ... & 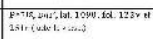 \\
\hline \multicolumn{3}{|l|}{ 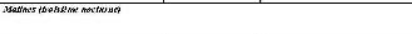 } \\
\hline 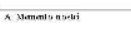 & $=n$ & \\
\hline 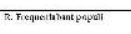 & Partia in & \\
\hline V. Moxque timentasta & 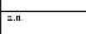 & \\
\hline 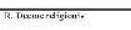 & 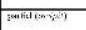 & \\
\hline
\end{tabular}

\begin{tabular}{|c|c|c|}
\hline 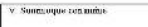 & $=n$ & \\
\hline R. cmisiat aces & 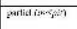 & - \\
\hline 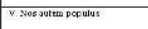 & $=.1$. & \\
\hline R. Constibithux & $\cdots$ & - \\
\hline 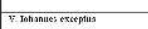 & in & \\
\hline \multicolumn{3}{|l|}{ Lovicat } \\
\hline 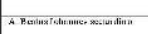 & samplats & \\
\hline 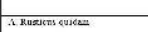 & Prent carasts & \\
\hline \begin{tabular}{|l|} 
Asse olstsmale \\
\end{tabular} & e.n & \\
\hline 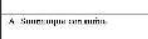 & $\approx$ & - \\
\hline 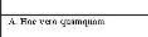 & somplict & \\
\hline \multicolumn{3}{|l|}{ 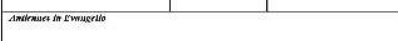 } \\
\hline 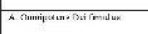 & smmplata & \\
\hline 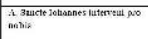 & $a n$ & \\
\hline 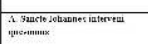 & campits & - \\
\hline \multicolumn{3}{|c|}{ 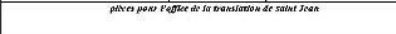 } \\
\hline \multicolumn{3}{|l|}{ Izpes } \\
\hline 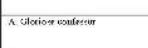 & an & 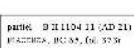 \\
\hline \multicolumn{3}{|l|}{ somotin } \\
\hline 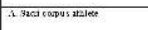 & s.n. & \\
\hline 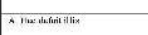 & $\cdots$ & \\
\hline 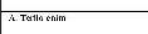 & an. & \\
\hline 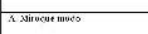 & $2 . n$ & \\
\hline 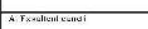 & $\cdots$ & \\
\hline
\end{tabular}




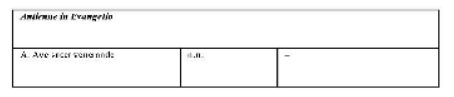

\section{NOTES}

1. Voir D. RUSSO et E. MAGNANI, «Le manuscrit 1 de la Bibliothèque municipale de Semuren-Auxois, provenant de l'abbaye de Saint-Jean de Réome (Moutiers-Saint-Jean) : programme pédagogique de recherche ", Bulletin du Centre d'études médiévales d'Auxerre, 12 (2008), p. 301-304 [http://cem.revues.org/document7212.html].

2. Cf. F. WORMALD, "Some illustrated manuscripts of the lives of the saints », Bulletin of the John Rylands Library, 35 (1952-1953), p. 248-266.

3. Avec des ajouts postérieurs en vers aux fol. or et $15 \mathrm{r}$ et de prières au fol. $50 \mathrm{r}$.

4. B. KRUSCH, «Vita Ihoannis abbatis Reomaensis autore Iona », in MGH, SS rer. Germ. in usum scholarum, 1905, p. 325 et n. 1.

5. Principales éditions du dossier hagiographique de saint Jean de Réome : P. ROUVIER (S.J., Le P.), Reomaus, seu Historia monasterii S. Joannis Reomaensis, in tractu Lingonensi, primariae inter gallica coenobia antiquitatis, ab anno Christi 425..., Paris, 1637, p. 1-23 et 70-71 (BHL 4426), p. 40-47 et extrait p. 73-74 (BHL 4429), p. 47-57 (BHL 4430) et p. 57-58 (BHL 4431) ; Société des Bollandistes, Acta Sanctorum, lanuarii II, Anvers, 1643, p. 856-862 (BHL 4427), p. 863-865 (BHL 4429), p. 865-868 (BHL 4430) et p. 856, n. 14 et 15 (BHL 4431); J. MABILLON, Acta Sanctorum ordinis S. Benedicti in Saeculorum Classes distributa. Saeculum I, Paris, 1668, p. 632-639 (BHL 4425) et p. 639-642 (BHL 4429); B. KRUSCH, "Vita Ihoannis abbatis Reomaensis autore lona », in MGH, Scriptores rerum merovingicarum, III, Hannovre, 1896, p. 505-517 (BHL 4424);ID., MGH, SS rer. Germ. in usum scholarum, 1905, p. 321-344 (BHL 4424) ; B. KRUSCH, Neues Archiv, 18 (1892), p. 615-616 (BHL 4428, 4428a et 4428b).

6. Dom A. WILMART, Codices Reginenses latini, t. 2, Cité du Vatican, 1945, p. 696-699. Le Vat. Reg. lat. 493 est un recueil hagiographique réuni à Saint-Germain d'Auxerre au XIIIe siècle, composé de pièces de différentes époques (XIe et XIIe siècles) et de provenances 
diverses. Le dossier de Jean de Réome relève d'un ensemble concernant les abbés de Cluny, notamment Maïeul (fol. 80r-105r). Voir D. IOGNA-PRAT, Agni Immaculati. Recherches sur les sources hagiographiques relatives à saint Maieul de Cluny (954-994), Paris, 1988, p. 51-52, 71-73 et 132-137.

7. Catalogue général des manuscrits des départements, Paris, 1879, t. 5, p. 88 (consultable en ligne: http://ccfr.bnf.fr/portailccfr/). Le manuscrit était un recueil de textes hagiographiques de différentes époques (Xe-XIe siècles) et contenait les vies des saints Grégoire, Jean de Réome, Samson, de sainte Walburge et la passion de saint Valentin.

8. Nous pourrions citer, en effet, outre le Semur 1, le Sacramentaire de Jumièges (PARIS, BnF, lat. 1141), en part. fol. 4r, vers 869/870; le Psautier de Saint-Germain-desPrés (PARIS, BnF, lat.11550), en part. fol. 8r, début du XIe siècle (?); le Graduel d'Albi (PARIS, BnF, lat. 776), en part. fol. 5r, XIe siècle ; le Missel de Saint-Denis (PARIS, BnF, lat. 9436), en part. fol. 13v, fin du XIe siècle.

9. Sur le principe, qualifié souvent de barbare, d'un entrelacs de formes et de figures voir, parmi d'autres: O. PÄCHT, Buchmalerei des Mittelalters, Eine Einführung, Munich, 1984; trad. fr. Paris, 1997, en part. chap. 2, «L'initiale ». Comparer à W. CAHN, Studies in Medieval Art and Interpretation, (recueil d'articles), Londres, 2000, en part. chap. 1, 5, 8 et 9 resp. «A Defense of the Trinity in the Cîteaux Bible», "Moses ben Abraham's Chroniques de la Bible », « Three Eleventh-Century Manuscripts from Nevers », «The Rule and the Book» et «Cistercian Book Illumination in Burgundy and Champagne ».

10. Comparer avec les procédés décrits par E. Vinaver, dans le champ des études relatives à la littéralité médiévale, À la recherche d'une poétique médiévale, Paris, 1970 ; en part. p. 9-10, à propos des deux «théories» qui s'affrontent dans ce champ, l'une dite «traditionaliste », l'autre « individualiste »: « (...) Dans l'un comme dans l'autre cas, l'œuvre se voit refuser le pouvoir de dire ce qu'elle est. Reflet d'une tradition lointaine ou amorce d'un idéal arbitrairement choisi, elle n'intéresse le chercheur qu'en vertu de quelque chose qui se situe hors d'elle."

11. En suivant une analyse phénoménologique, nous dirions que cet espace, défini par le livre précieux, par sa présence même, est tout ensemble un « site » logique et un « site » ontique, pour l'affirmation d'une forte identité monastique, dans l'exemple suivi du Semur 1, à ce moment précis de l'histoire de la communauté. Pour une discussion philosophique de ces concepts, cf. F. WAHL, Du perçu, Paris, 2007 ( (Ouvertures »).

\section{INDEX}

Mots-clés : chant, manuscrit 